\title{
Intermediate filaments: primary determinants of cell architecture and plasticity
}

\author{
Harald Herrmann, ${ }^{1}$ Sergei V. Strelkov, ${ }^{2}$ Peter Burkhard, ${ }^{3}$ and Ueli Aebi ${ }^{4}$
}

\author{
${ }^{1}$ Group Functional Architecture of the Cell, B065, German Cancer Research Center, Heidelberg, Germany. \\ 2Department of Pharmaceutical Sciences, Katholieke Universiteit Leuven, Leuven, Belgium. ${ }^{3}$ The Institute of Material Science, \\ University of Connecticut, Storrs, Connecticut, USA. ${ }^{4}$ M.E. Müller Institute for Structural Biology, Biozentrum, University of Basel, Basel, Switzerland.
}

\begin{abstract}
Intermediate filaments (IFs) are major constituents of the cytoskeleton and nuclear boundary in animal cells. They are of prime importance for the functional organization of structural elements. Depending on the cell type, morphologically similar but biochemically distinct proteins form highly viscoelastic filament networks with multiple nanomechanical functions. Besides their primary role in cell plasticity and their established function as cellular stress absorbers, recently discovered gene defects have elucidated that structural alterations of IFs can affect their involvement both in signaling and in controlling gene regulatory networks. Here, we highlight the basic structural and functional properties of IFs and derive a concept of how mutations may affect cellular architecture and thereby tissue construction and physiology.
\end{abstract}

\section{Introduction}

Human cells contain three principal filament systems: microfilaments (MFs), microtubules (MTs), and intermediate filaments (IFs). They form a dynamic cytoskeleton that mechanically integrates cellular space (1-3). Actin and tubulin, the proteins constituting MFs and MTs, respectively, are highly conserved globular proteins. In humans, they are both expressed in the form of three major subtypes termed $\alpha, \beta$, and $\gamma$. In contrast, the members of the IF protein family, which is encoded by 70 genes, have very different primary amino acid sequences but a common domain organization (4). In addition, an increasing number of alternative splice forms of individual IF proteins have been discovered recently (5). IF proteins are differentially expressed during embryonic development, in parallel to distinct routes of differentiation, indicating that they have distinct tissue-specific functions $(6,7)$. Hence, in epithelial cells, a highly diverse group of keratins is expressed; vimentin is a hallmark of mesenchymal, endothelial, and hematopoietic cells; the extra-sarcomeric cytoskeleton of myoblasts consists mainly of IFs, containing desmin, synemin, and syncoilin; and neuronal cells are characterized by IFs coassembled from the neurofilament triplet proteins and $\alpha$ internexin $(8,9)$. A further contrast to MFs and MTs is that higher metazoan interphase cells harbor two distinct IF systems in parallel, one in the nucleus and one in the cytoplasm (Figure 1). The nuclear IF system in interphase cells constitutes a meshwork-like lamina of partially heterogeneous and independently organized filaments made of lamin $A$, lamin $C$, lamin $B_{1}$, and lamin $B_{2}(10)$. The lamina is apposed to the nuclear envelope and integrates inner nuclear membrane proteins, nuclear pore complexes, and heterochromatin into a functional interface $(11,12)$. The nuclear IF network is directly coupled to the cytoplasmic IF system via plectin and interacting inner and outer nuclear membrane proteins, such as nesprin- $3 \alpha$ and Sun proteins (13). During mitosis, the lamin system is disassembled through the action of mitotic kinases, whereas the cytoplasmic IF system is depolymerized only in some cell types $(14,15)$. Notably, a

Conflict of interest: The authors have declared that no conflict of interest exists.

Nonstandard abbreviations used: IF, intermediate filament; $K$, keratin; MF, microfilament; MT, microtubule; SHC, sequence homology class; ULF, unit-length filament.

Citation for this article: J. Clin. Invest. 119:1772-1783 (2009). doi:10.1172/JCI38214. specific fraction of the lamins, lamin $\mathrm{B}_{2}$, is reorganized and incorporated into a supportive structure for the mitotic spindle that is termed the spindle matrix (16).

The proteins that constitute MTs, MFs, cytoplasmic IFs, and nuclear IFs are able to self-assemble in vitro (Figure 2). $\alpha$ - and $\beta$-tubulin heterodimers form 25-nm-diameter MTs made from 13 protofilaments of identical orientation, whereas actins assemble into 9-nm-diameter MFs made of two right-handed long-pitch helical strands of globular subunits, with an axial stagger of half a subunit relative to each another $(17,18)$. Both MFs and MTs are topologically closed polymers, exhibiting a distinct polarity with a fast- and a slow-growing end. This polarity is essential for their use as tracks for motor proteins in unidirectional transport. In contrast, cytoplasmic IF proteins form apolar, smooth, and flexible filaments, with an approximate diameter of $10 \mathrm{~nm}$, i.e., the filaments are "intermediate" in diameter between that of MTs and MFs, hence the name "intermediate filaments." Lamins assemble in vitro into filaments somewhat distinct from those formed by the cytoplasmic IF proteins; for example, they exhibit a pronounced 24-nm axial beading repeat (Figure 2). Slowly, but definitely, in vitro assembled lamin filaments form, probably by lateral association, extensive paracrystalline fibers (Figure 2) $(10,19)$. In further stark contrast to MFs and MTs, for which general depolymerizing compounds often used in cancer chemotherapy are known, as of yet no general inhibitors of assembly have been described for IFs (20-22).

In searches for IF proteins in species other than mammals, it became apparent that IFs are hallmark cytoskeletal entities of all metazoan organisms. Hence, IF proteins are found in simple animals, such as the sweet water sponge Hydra and the nematode Caenorbabditis. IFs are, however, in contrast to MFs and MTs, not expressed in plants and fungi. Their absence may correlate with the fact that the body plan of plants and fungi depends on mechanical support provided by external cell walls. Similarly, insects do not harbor cytoplasmic IF proteins; however, they do have a nuclear IF system made from authentic lamins.

In higher vertebrates, the number of genes encoding IF proteins increased considerably during evolution. However, already lower invertebrates such as the nematode Caenorhabditis elegans express more than ten IF proteins, several of which are essential for life (23-26). In humans, keratins represent the major subgroup of IF 

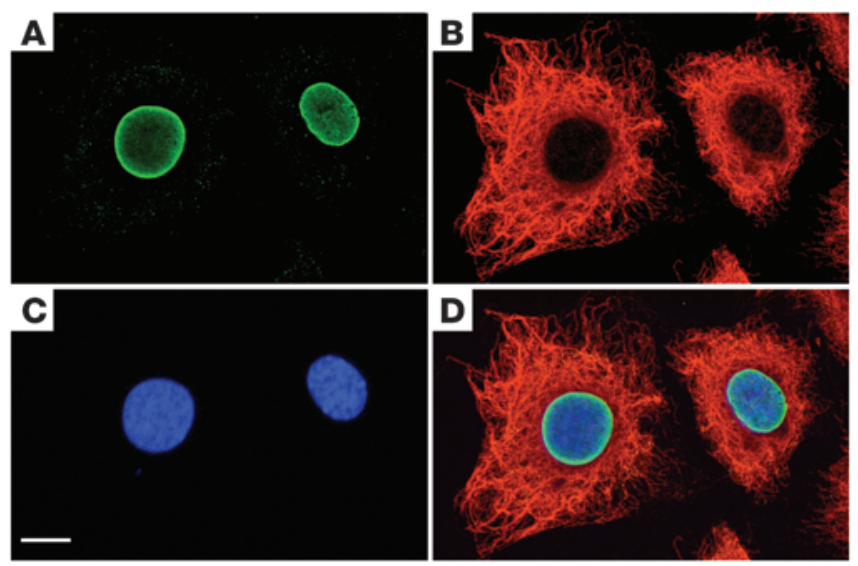

Figure 1

Cells from higher metazoan organisms harbor two distinct IF systems. Indirect immunofluorescence staining with antibodies specific for $(\mathbf{A})$ lamin A and (B) vimentin indicates that lamins are concentrated at the inner nuclear membrane within the nucleus, whereas vimentin forms a complex network within the cytoplasm that extends from the cell periphery immediately to the outer nuclear membrane. (C) DNA has been stained with the DNA-specific dye DAPI (blue). (D) Merged image of $\mathbf{A}-\mathbf{C}$. Scale bar: $10 \mu \mathrm{m}$.

proteins and are encoded by 54 genes, about half of them being expressed by trichocytes, the specialized epithelial cells from which hair, nails, and similar appendages are generated. Thirteen genes encode mesenchymal, muscle, lens-specific, and neuronal IF proteins, and three genes encode the four nuclear lamins (27). Following detailed amino acid comparisons in higher vertebrates, researchers have grouped IF proteins into six sequence homology classes (SHCs) (also known as "types"): SHC1 comprises the acidic keratins; SHC2 comprises the basic keratins; SHC3 includes vimentin, desmin, glial fibrillary acidic protein (GFAP), and peripherin; SHC4 harbors the neurofilament triplet proteins together with $\alpha$ internexin and nestin; SHC5 represents the lamins; and SHC6 consists of the two lens-specific proteins, beaded filament structural protein 1 (Bfsp1; also known as filensin) and Bfsp2 (also known as phakinin and CP49) (28).

Although functional inactivation of genes encoding IF proteins, such as keratins, vimentin, and desmin, is mostly not embryonal lethal in mice, their absence has been shown to have a considerable impact on cellular and tissue physiology. For instance, mice lacking a functional vimentin gene are apparently normal, but primary fibroblasts derived from these animals exhibit a substantial reduction in stiffness (29). Moreover, these cells display reduced mechanical stability, motility, and directional migration. In addition, the spatial organization of the focal adhesion proteins is disturbed. As a result, the ability of these cells to repair a wounded fibroblast monolayer is severely impaired (29). Correspondingly, wounds inflicted upon vimentin-deficient embryos heal much more slowly than those in control embryos (30). This effect has been attributed to a failure of mesenchymal contraction at the embryonic wound site (30). Another way in which a lack of vimentin affects cellular and tissue physiology is that the performance of both peripheral blood mononuclear cells and endothelial cells in transendothelial migration is greatly diminished in vimentin-deficient mice, indicating that vimentin IFs are important for lymphocyte adhesion and transmigration through the endothelium (31).
In this Review, we discuss how disease-causing mutations in IF protein-encoding genes possibly affect the structure and assembly of IF proteins. Moreover, we present a view - with the muscle IF system as a paradigm - of how the change in basic IF properties may impact cell architecture and plasticity, and, thereby, eventually tissue function.

\section{A structural blueprint for IF proteins}

In contrast to actin and tubulin, which are globular proteins, IF proteins are fibrous. In further contrast to MFs, MTs, and most other cytoskeletal proteins, IFs, together with some associated proteins such as desmoplakin and plectin, exhibit a pronounced stability in buffers containing high concentrations of detergent and salt, indicating that the individual subunits very tightly associate through both ionic and hydrophobic interactions (32-34). The molecular blueprint of IF proteins exhibits a tripartite structural organization; they consist of a central $\alpha$-helical rod domain, which is flanked by non- $\alpha$-helical amino-terminal head and carboxyterminal tail domains (Figure 3A) $(35,36)$. Within the $\alpha$-helical rod domain, amino acids are regularly organized in heptad repeats, such that usually every first and fourth amino acid side chain is hydrophobic, thereby generating a hydrophobic seam, which in turn drives the association of like molecules into a coiled coil (37, 38). Hence, the principal structure of IF proteins is an $\alpha$-helical rod that is $45 \mathrm{~nm}$ in length in cytoplasmic IF proteins and $7 \mathrm{~nm}$ longer in nuclear IF proteins (32). Two $\alpha$-helical rods are able to associate in a parallel, unstaggered fashion to form a left-handed superhelix, i.e., a coiled-coil dimer. In contrast to all other IF proteins, keratins form obligate heterodimers, containing an acidic SHC1 keratin and a basic SHC2 keratin $(39,40)$.

On the basis of extensive comparisons of the amino acid sequence of many IF proteins and a correlated structure prediction, it was concluded and generally accepted that the heptad repeat pattern of the $\alpha$-helical rod is interrupted by three linker domains, creating four sub-helices that have been termed coil 1A, coil 1B, coil 2A, and coil $2 \mathrm{~B}$ (Figure $3 \mathrm{~A})$. The number of amino acids found in the individual helices is strictly conserved in the various types of IF proteins except for coil 1B, which is 42 amino acids (or six heptads) longer in the nuclear lamins than in vertebrate cytoplasmic IF proteins (143 versus 101 amino acids). Notably, cytoplasmic IF proteins of lower invertebrates also exhibit the longer coil $1 \mathrm{~B}$ version, underlining their evolutionary relationship to lamins. The length of the other coils is identical in all IF proteins: coil $1 \mathrm{~A}$ has 35 amino acids, coil 2A has only 19 amino acids, and coil 2B has 115 amino acids (1). In the middle of coil 2B, a discontinuity in the heptad repeat pattern, a so-called stutter, is consistently found in all IF proteins.

Vimentin-like IF proteins differ from lamins and keratins, as they have an $\alpha$-helical segment of 20 amino acids that precedes coil $1 \mathrm{~A}$ and may even form a coiled coil (41). The linker domains between the coils are referred to as $\mathrm{L} 1$ (which connects coil $1 \mathrm{~A}$ and coil 1B), L12 (which connects coil 1B and coil 2A), and L2 (which connects coil $2 \mathrm{~A}$ and coil $2 \mathrm{~B}$ ). The length of the individual linkers is not strictly conserved and their structure is not completely clear. However, in lamins, L1 is always 11 -amino acids long, and in every IF protein, L2 is eight-amino acids long (Figure 3B) (8, 42). Although in silico structural prediction served well for some time to describe the structure of IF proteins, our recent crystallographic studies (our unpublished observations) indicate that certain adjustments to the earlier picture of the second half of the rod are needed. The segments previously termed coil 2A, L2, 
MT

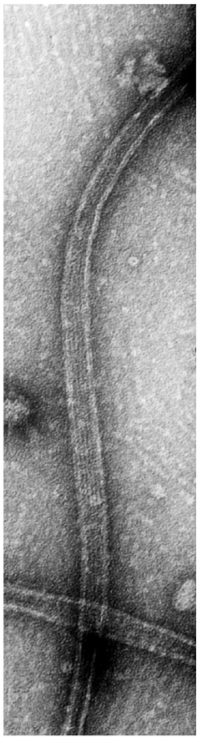

MF

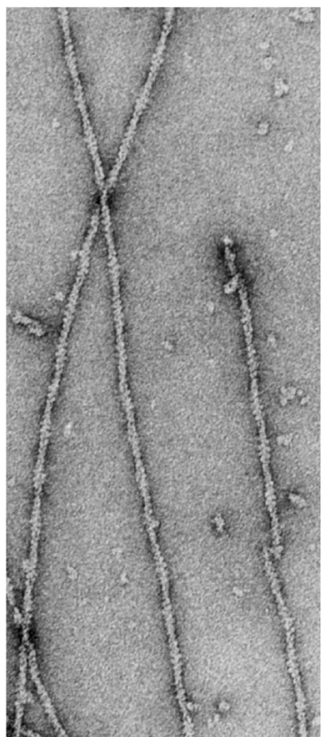

Cyt-IF

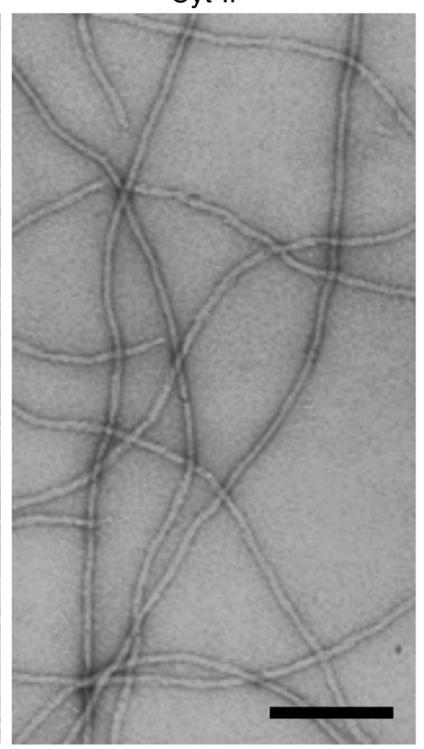

Lamin-IF

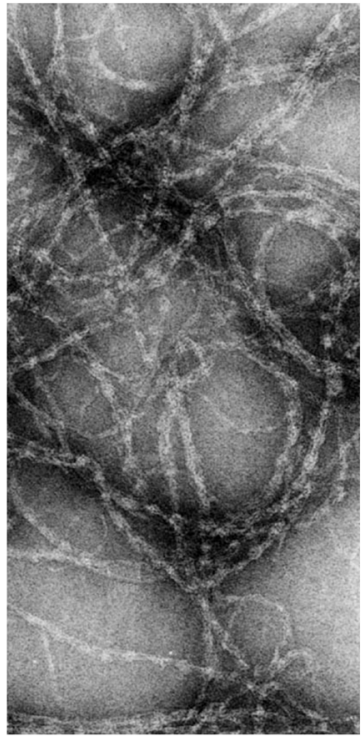

Lamin-Pc

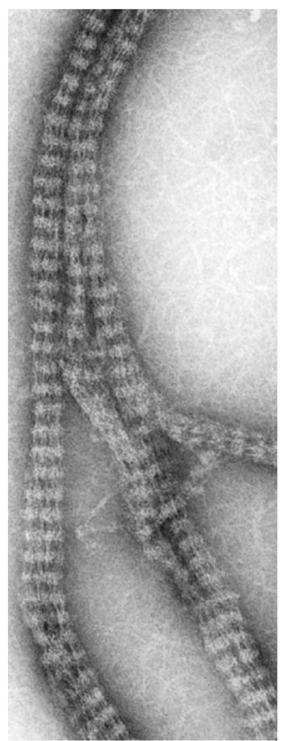

Figure 2

Ultrastructural analysis of the principal filamentous elements of metazoan cells after in vitro reconstitution. Negatively stained preparations of MTs, MFs, vimentin IFs (Cyt-IFs), lamin A filaments (Lamin-IFs), and paracrystalline lamin A fibers (Lamin-Pc), as visualized by transmission electron microscopy. Scale bar: $100 \mathrm{~nm}$.

and coil 2B (and now designated coil 2) constitute a continuous coiled coil of 142 amino acids, with two short segments in which the helices run in parallel. The short parallel bundle (Figure $3 \mathrm{~B}$ ) is formed by the segments previously called coil $2 \mathrm{~A}$ and L2, and it is followed by a normal coiled-coil segment that harbors the stutter, a local unwinding of the otherwise intact coiled coil that causes the two $\alpha$-helices to become nearly parallel at this site $(43,44)$. The length of coil 2 in every IF protein is approximately $21.5 \mathrm{~nm}$. In contrast to the high structural similarity in coil 2 of vimentin-like IF proteins (the SHC3 and SHC4 IF proteins), keratins (SHC1 and SHC2 IF proteins), and lamins (the SHC5 IF proteins), these three groups of IF proteins exhibit substantial structural differences in the organization of coil 1 (i.e., the rod segment harboring coil 1A, linker L1, and coil 1B) and hence constitute three groups of IF proteins with distinct structural differences (Figure 3B).

The above building plan of IF proteins (Figure 3A) is also "diagnostic," providing a tool to analyze the primary sequences of newly identified proteins in order to find out if they belong to this family (45). Hence, as this structural plan is not followed in crescentin, a Caulobacter crescentus protein reported to be a prokaryotic IF-like protein (46), it probably is not an "IF ancestor." Consistent with this idea, no homologs of crescentin have been found in other bacteria. Interestingly, however, bacterial non-IF proteins with a central coiled coil have been identified recently to constitute cytoskeletal networks, underlining the efficiency of fibrous proteins forming two-stranded $\alpha$-helical coiled coils as versatile basic elements of structural scaffolds (47). Moreover, coiled-coil-forming non-IF proteins, such as myosins, tropomyosins, kinesins, plakins, and transcription factors, are also frequently found in many eukaryotic organisms.

A major advance in defining the structural organization of IF proteins has been accomplished through comparison of numerous individual protein sequences obtained by screening cDNA libraries of various species. Due to the extremely high conserva- tion of the building plan of practically all known IF proteins (Figure $3 \mathrm{~B}$ ), some robust structure predictions became feasible (2). Comparing the sequences of many different IF proteins, especially over a wide range of species, two amino acid-sequence stretches, the so-called IF-consensus motifs, became evident at either end of the $\operatorname{rod}($ Figure $3 \mathrm{~A})$ : one encompassing the amino-terminal end of coil $1 \mathrm{~A}$ and one marking the end of coil $2 \mathrm{~B}$. The latter IF-consensus motif, a stretch of 10 amino acids (TYRKLLEGEE), is nearly identical in proteins as distinct as the Hydra nuclear lamin and human hair keratins (48). Similarly, the IF-consensus motif in coil $1 \mathrm{~A}$ is extremely well conserved for individual IF proteins, such that the vimentins from species as diverse as primitive fish and humans are nearly identical over a stretch of 20 amino acids, including the absolutely conserved sequence LNDR, which is present in nearly every IF protein $(42,49)$.

Considering such conservation of amino acid sequence among diverse species, it comes as no surprise that mutations in these motifs interfere drastically with the function of IF proteins. This is highlighted by the discovery that inherited mutations in keratinencoding genes cause human skin fragility diseases and the experimental verification in transgenic mice that such mutations have a devastating impact on proper skin architecture and function (50, 51). Although disease-causing mutations in the coil 1A-consensus motif of keratins do not prevent extended in vitro filament assembly, arguing that they may interfere at the level of the spatial organization of the keratin cytoskeleton, an experimental mutation introduced into this consensus motif of vimentin inhibits in vitro assembly completely (52-55).

\section{Atomic structures of the coiled-coil segments}

The two key questions to be answered if we are to understand the basic principles of IF biology are what is the structure of an IF dimer and, eventually, an entire IF at atomic detail, and how do particular 

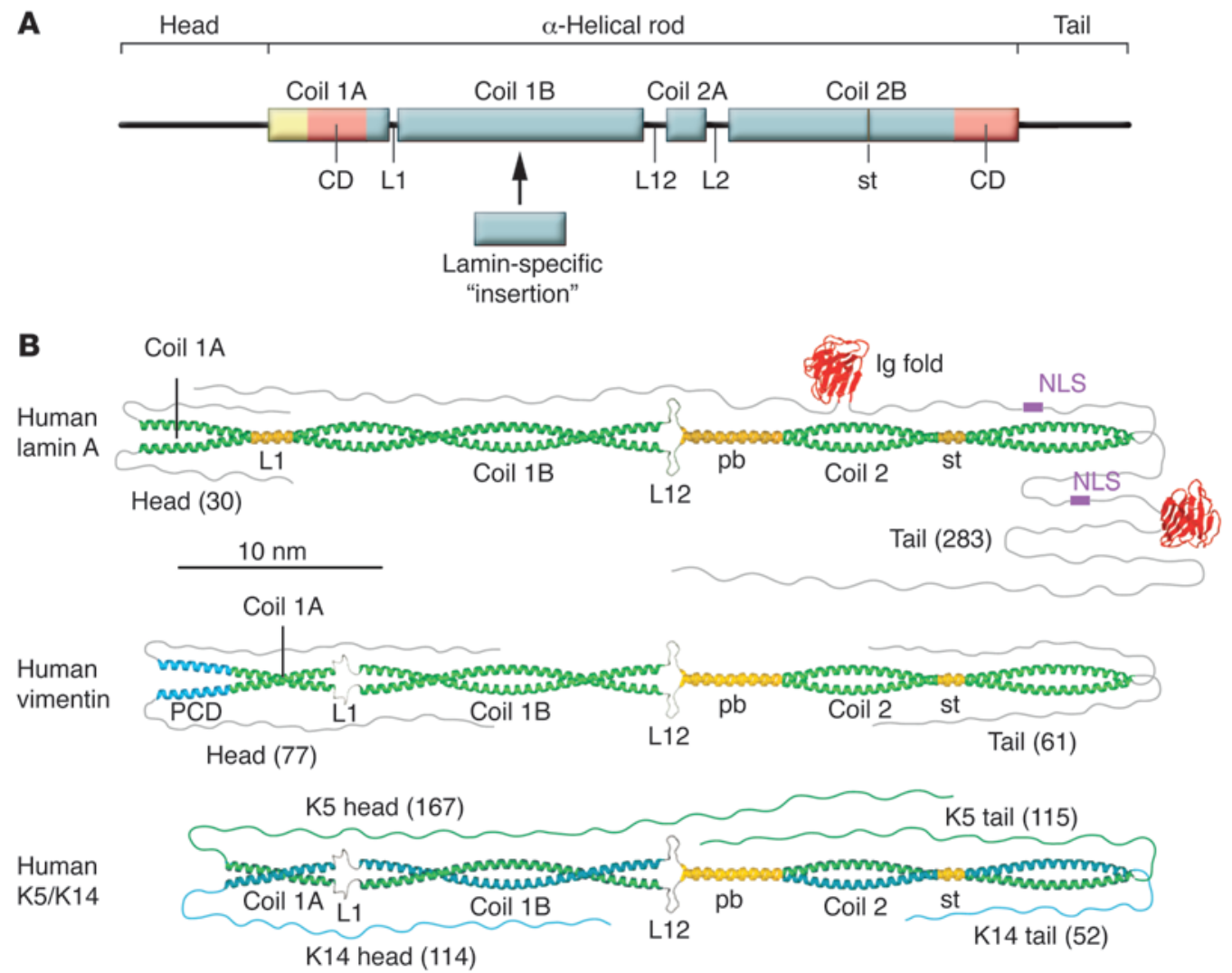

\section{Figure 3}

Molecular organization of IF proteins. (A) Schematic representation of the structural organization of an IF molecule. Blue boxes represent segments; orange boxes represent IF-consensus motifs (CD); and the yellow box represents an $\alpha$-helical pre-coil domain only found in vimentin-like IF proteins. L1, L12, and L2 are linker segments. As L2 is predicted to form an $\alpha$-helical segment, coil $2 A$ and linker L2 are designated as paired bundle $(\mathrm{pb})$ in B. "Head" and "tail" represent non- $\alpha$-helical domains. The stutter (st) indicates a discontinuity in the heptad repeat pattern of coil 2B (43). The blue box designated lamin-specific "insertion" highlights a position in which an additional $\alpha$-helical segment is found in lamins and lower invertebrate cytoplasmic IF proteins. (B) Model of the dimeric structures exhibited by human lamin A, vimentin, and the epidermal pair K5/K14. Vimentin exhibits a pre-coil domain (PCD). Moreover, a special feature of nuclear lamins, as opposed to cytoplastic IF proteins, is that linker L1 in lamins is likely to be $\alpha$-helical. The numbers in parentheses indicate the number of amino acids in the respective non- $\alpha$-helical domains. NLS, nuclear localization signal. B is adapted from Nature reviews molecular cell biology (8).

IF proteins assemble into filaments? To answer the first question, researchers attempted to solve the molecular organization of a coiled-coil vimentin dimer by X-ray crystallography. However, as it became clear that the entire dimeric rod would not easily crystallize, a "divide-and-conquer" strategy was developed, and subfragments of the rod were subjected to crystallization (56). As a first step, the structure of a 28-amino acid segment of coil 2B of human vimentin, including its consensus motif, was solved using crystals formed by a chemically synthesized peptide (48). Most interestingly, both glutamic acid 396 and arginine 401, which form an inter-helical salt bridge in the crystal, are highly conserved among all IF proteins at the corresponding positions in coil 2B. Thus, it is perhaps not surprising that mutations of the homologous amino acids in keratins, desmin, GFAP, and lamins cause disease (48). According to the specific tissue localization of the proteins, mutations that affect the salt bridge in epidermal keratins lead to blistering diseases, in desmin they lead to muscular dystrophy, and in GFAP they lead to Alexander disease (57-64). In contrast, mutation of lamin A arginine 377 , the arginine residue that corresponds to arginine 401 in vimentin, to either leucine or histidine gives rise to limb-girdle muscular dystrophy type 1B and Emery-Dreifuss muscular dystrophy (65-67). Moreover, the R377H mutation can also cause dilated cardiomyopathy type A (68). Most notably, mutations in either amino acid of this inter-helical salt bridge of lamin A represent one of the three major hotspots for laminopathic disease mutations (Giselè Bonne, personal communication).

The initial crystallization studies were followed by the determination of the atomic structure of coil $1 \mathrm{~A}$ and the second half of coil 2, then called coil 2B, for both vimentin and lamin A $(43,44)$. Whereas coil 2B was shown to form bona fide dimeric complexes, coil $1 \mathrm{~A}$ remained monomeric in solution (56). Even more surprising, coil $1 \mathrm{~A}$ was also obtained as a monomer in the crystals that formed at high-protein concentration, although it assumed the left-handed helical supertwist necessary for coiled-coil formation. Nevertheless, in the full-length protein, coil $1 \mathrm{~A}$ is not isolated but embedded in a distinct structural context, and larger fragments containing the head domain in addition to coil 1A behave, under certain in vitro conditions, like a dimeric coiled coil (56). A 20amino acid segment of the head domain that precedes coil $1 \mathrm{~A}$ is probably important for the observed dimerization of this extended 
Phase 1

Lateral association of tetramers into ULFs

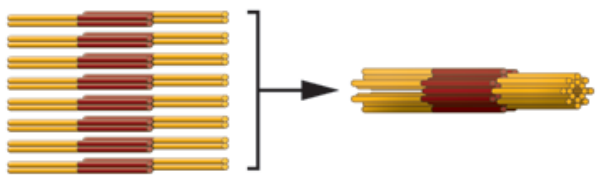

Phase 2

Longitudinal annealing of ULFs and filaments

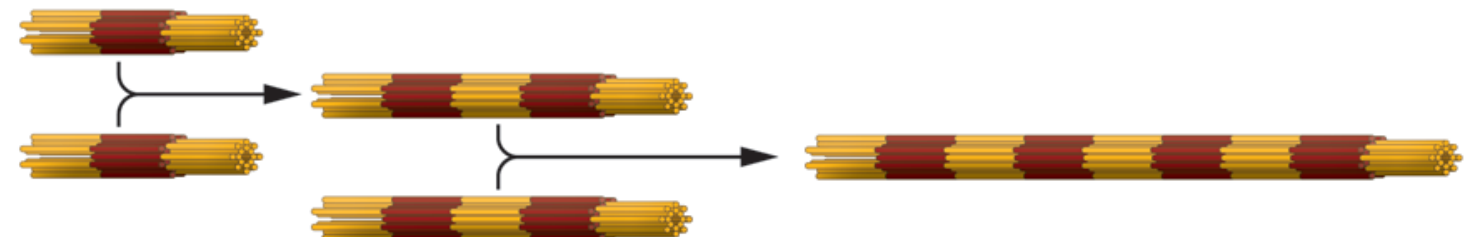

Phase 3

Radial compaction of extended filaments

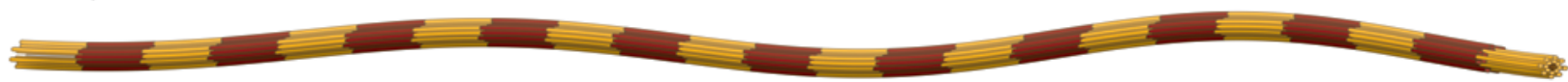

Figure 4

Schematic representation of the three major phases of cytoplasmic IF assembly. In phase 1, eight tetrameric subunits made from two antiparallel, half-staggered coiled-coil dimers associate laterally to form ULFs after initiation of assembly. Every single vimentin molecule is represented by one cylinder; coil 1 of each molecule is colored dark red, and coil 2 is colored yellow. In phase 2, ULFs and short filaments longitudinally anneal to other ULFs and filaments. In phase 3, after about ten minutes of assembly, filaments have radially compacted to a diameter of approximately $11 \mathrm{~nm}$. Adapted with permission from Journal of biological chemistry (80).

coil 1A fragment; according to sequence prediction, this segment may form an $\alpha$-helix and possibly even a coiled coil (Figure 3B) (38, 41,42 ). In addition, the head domains of IF proteins (vimentin, lamins, and keratins) may help to stabilize the dimeric state.

Crystallized coil 2B exhibits very much the same structural fold in lamin A and vimentin, and it is expected that keratins will exhibit a very similar structure once they are crystallized, due to their conserved amino acid sequence organization in the corresponding segment. Therefore, coil 2 represents a truly conserved structural feature that all IF proteins exhibit in common (Figure 3B). The functional meaning for this similarity is at present not clear. However, as most rules do not come without an exception, the single lamin of the nematode Caenorhabditis elegans lacks two heptads in coil 2 (69). Nevertheless, this protein assembles into IF-like filaments and paracrystalline fibers (70-74).

A dramatic evolution in the structure of IF proteins is reflected by their non- $\alpha$-helical domains, i.e., the "heads" and "tails" (75). These can be as short as 15 amino acids, as found for the tail of human keratin 19 (K19), or as long as 1,300 amino acids, as in the human neuronal IF protein nestin. Moreover, the nestin tail exhibits a high degree of sequence variation, even between related species such as rat and human (76). Hence, the nestin-encoding gene is in dynamic development in vertebrate evolution. The size of the head domain also varies widely and can be as long as 167 amino acids, as in K5 (Figure 3B). In contrast, the nestin head domain is unusually short, with only six amino acids. The head domain is generally critical for formation of IFs, as headless IF proteins do not assemble beyond the coiled-coil stage (42). Hence, the absence of a regular head domain in an IF protein such as nestin points to the fact that it needs to form a complex with a "headed" partner, such as vimentin, in order to be incorporated into an IF. Whether nestin and vimentin form heterodimers or whether nestin dimers associate with vimentin dimers before incorporating into IFs is not completely clear at present (77).

\section{The IF assembly mechanism: three distinct assembly groups}

The three major structural IF protein groups discussed earlier (see A structural blueprint for IF proteins) also give rise to three more or less distinct assembly groups. Hence, lamins, vimentin-like IF proteins, and keratins will not coassemble into mixed IFs; rather, they completely segregate into distinct structures, even within the same cell (34). Here, we concentrate our discussion on the in vitro assembly process of the cytoplasmic IF proteins vimentin and desmin, because it is the best understood from the biophysical characterization of the essential assembly intermediates up to the mathematical modeling of the assembly kinetics (78-81). The assembly reaction can be dissected into three major steps (Figure 4). In preparation for assembly, IF proteins reconstituted from $8 \mathrm{M}$ urea into low-salt buffer organize into relatively uniform tetrameric complexes of approximately half-staggered, antiparallel coiled-coil dimers; these are entirely soluble. In the first phase of assembly, which is initiated by increasing the ionic strength of the buffer, an average of eight tetramers rapidly associate laterally into unit-length filaments (ULFs). In the second phase, ULFs longitudinally anneal to form short filaments, and filament growth proceeds further by end-to-end association of filaments. In the third phase, there is a highly cooperative radial compaction of the nascent, "open," approximately 16-nm-diameter filaments into wide, mature, approximately 11-nm-diameter 
A

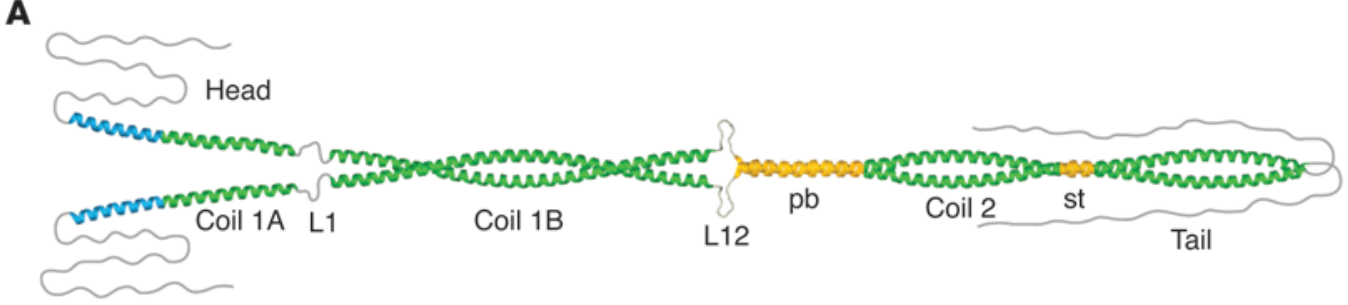

B
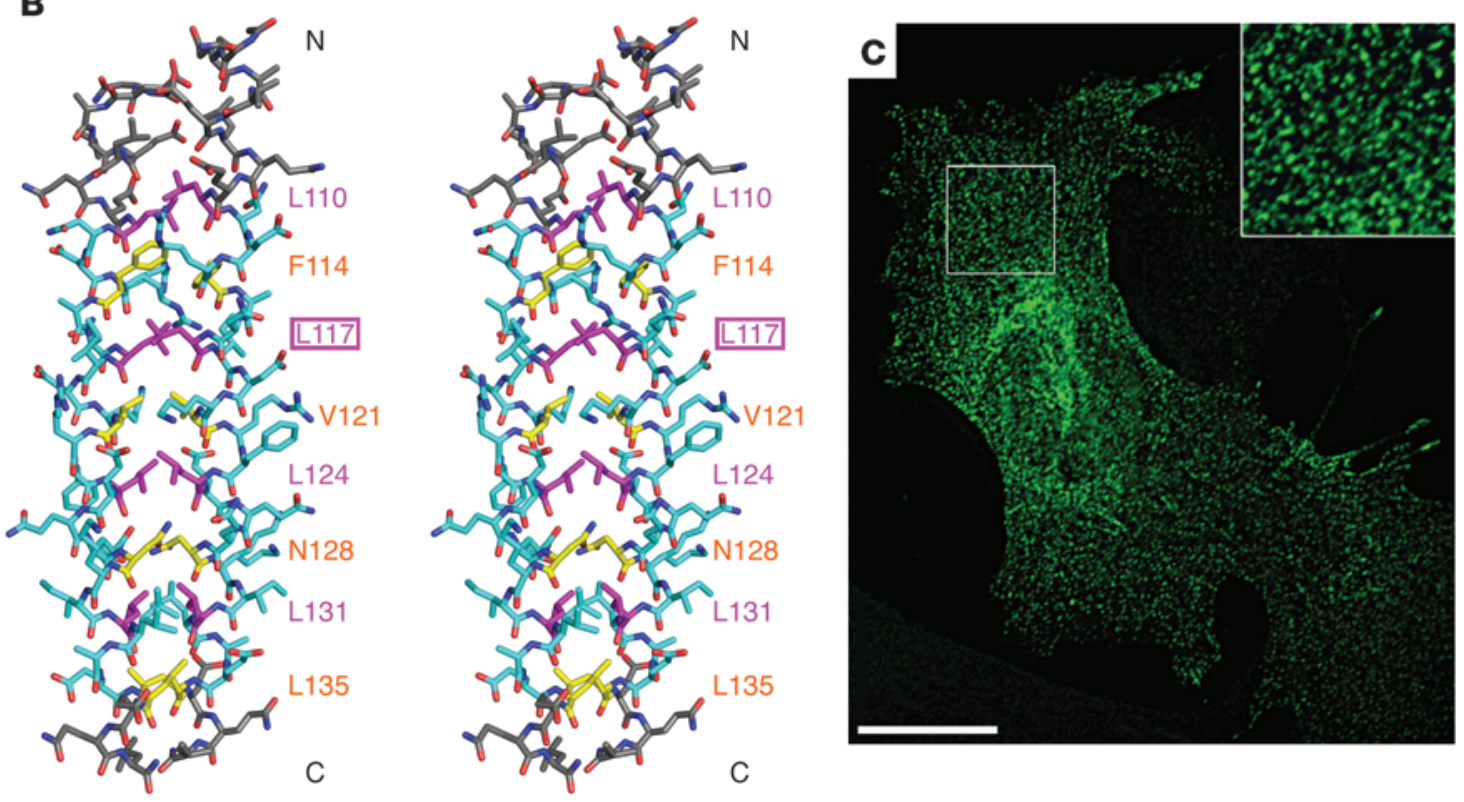

Figure 5

Impact of a point mutation in vimentin coil $1 \mathrm{~A}$ on filament elongation both in vitro and in vivo. (A) Hypothetical model depicting that coil $1 \mathrm{~A}$ may have a tendency to open up with the authentic tyrosine 117, shown in extreme conformation. (B) Side stereo view of the atomic structure of coil $1 \mathrm{~A}$ from the vimentin mutant $\mathrm{Y117L}$, exhibiting a bona fide coiled coil. Residues bounded by the first and the last knobs-into-hole interaction are colored in cyan. Residues at a positions are shown in yellow and those at $d$ positions are shown in magenta. The $\mathrm{N}$ terminus is marked $\mathrm{N}$, and the $\mathrm{C}$ terminus is marked $\mathrm{C}$. The residue types and numbers of the core positions are indicated. (C) Transfection of mouse embryonic fibroblasts derived from vimentin-knockout mice (130) with the vimentin mutant $Y 117 \mathrm{~L}$, followed by indirect immunofluorescence microscopy with antibodies specific for human vimentin, revealing dot-like structures exclusively. Note the regular shaped particles that on occasion are lined up, possibly being situated on a fibrillar structure such as a MT (inset). Scale bar: $20 \mu \mathrm{m}$. A is reproduced with permission from EMBO journal (43). B and C are reproduced with permission from Journal of molecular biology (92).

IFs $(82,83)$. All assembly steps very likely engage a number of complex molecular rearrangements, such that the flexibility of individual subdomains within the rod domain is important for successive interactions (83).

A major determinant of the annealing reaction, in which the ends of two filaments mediate the longitudinal fusion of two ULFs or short filaments, may reside in the structural state of the two end segments of the rod. Taking the comparatively weak interaction between two coil 1A chains as an argument, it was previously suggested that the two-stranded coiled coil of the vimentin dimer may partially open in its coil $1 \mathrm{~A}$ region to facilitate longitudinal annealing, followed by a lock-in-type reaction, thereby generating stable joints between annealed segments $(43,84)$. These interactions may be directly affected by phosphorylation of various serine residues in the head domain of vimentin, which can lead to the fragmentation and dissociation of vimentin IFs (85-87). Consistent with the idea that the conserved end segments of the rod are important for elongation, chemical cross-linking experiments have revealed an overlap-type interaction of the amino- and carboxyterminal domains of the vimentin rod (1). These types of interactions may represent major molecular contacts mediating the longitudinal annealing of ULFs and short filaments.

\section{Unifying principles in IF structure and assembly?}

As expected given that there are three distinct assembly groups (discussed earlier in The IF assembly mechanism: three distinct assembly groups), the overall structure of different IFs varies considerably. Therefore, the assumption that there exists just one type of "10-nm" IF may not be justified by the data obtained from tissue samples. Actually, the diameter of IFs, as measured in ultrathin sections from various tissues, was early on referred to as $7-11 \mathrm{~nm}$, indicating high structural variability between the various IFs assembled from distinct IF proteins (88). Note that the cross-sectional area of an 11-nmdiameter filament is twice that of a 7-nm-diameter IF, and therefore it harbors twice as many subunits as the thinner filament. Most likely, the resistance of an IF to mechanical stress increases with the number of subunits within a filament, and therefore this structural polymorphism may be of functional importance. 
A
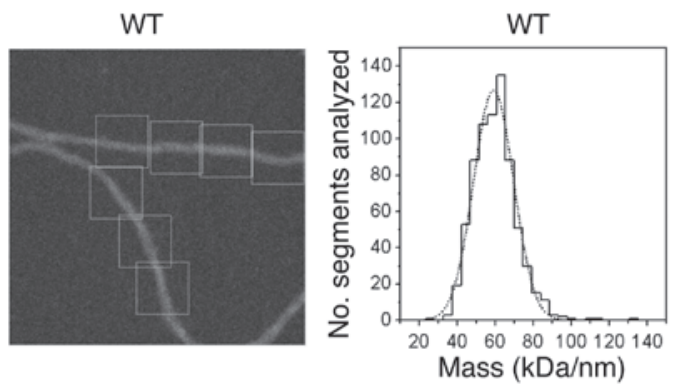

B

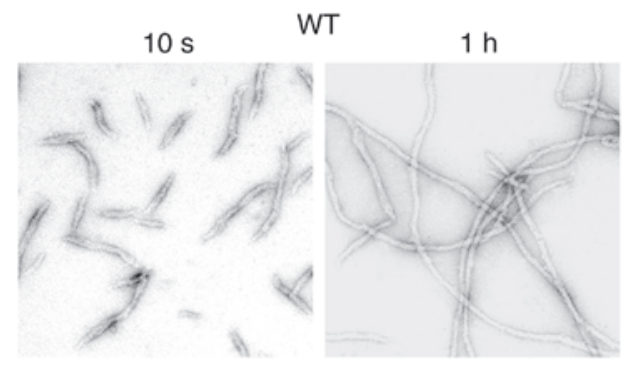

C

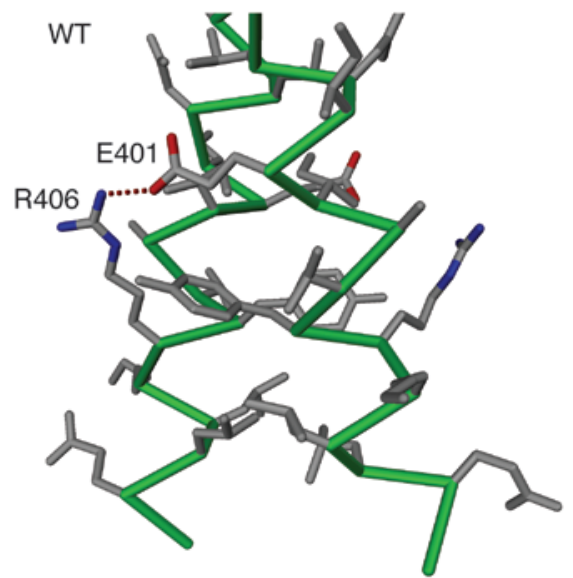

A360P
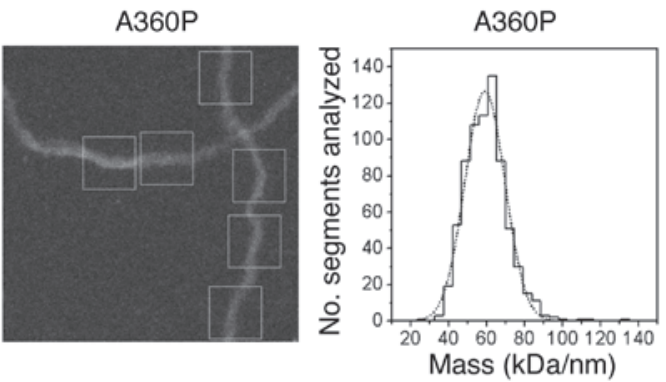

R406W
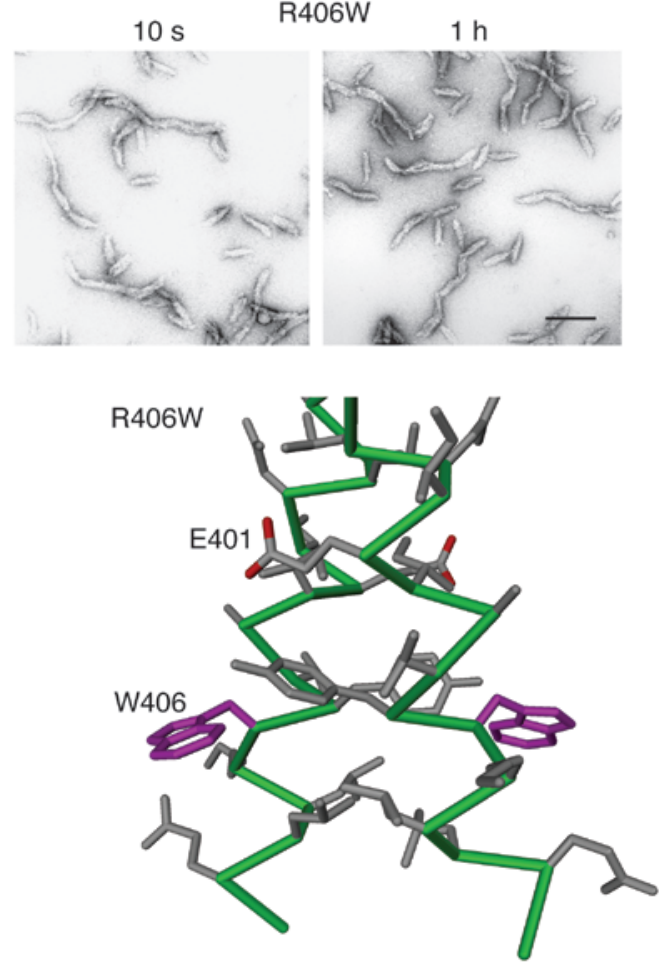

Figure 6

Molecular characterization of the desmin mutants A360P and R406W. (A) Mass measurement of assembled wild-type desmin (WT) filaments in comparison with filaments formed by the mutant A360P. Nonstained, lightly fixed filaments were analyzed by scanning transmission electron microscopy and visualized in a dark field mode (first and third panels from the left). The rectangles indicate the areas used for the quantification of mass. One side of the square is 60 -nm long. The molecular mass of individual filaments was determined along the various individual filaments and plotted as $\mathrm{kDa} / \mathrm{nm}$ into a histogram (second and fourth panels from the left). The abscissa shows mass in $\mathrm{kDa} / \mathrm{nm}$; the ordinate shows the number of segments analyzed. (B) Filament assembly of wild-type desmin in comparison to that of the mutant R406W, as depicted by electron microscopy of negatively stained samples obtained at 10 seconds and 1 hour. Scale bar: $100 \mathrm{~nm}$. (C) C-terminal region of the wild-type desmin dimer (left panel) compared with the R406W mutant (right panel). The mutated amino acid is shown in magenta. The change of arginine (R406) to tryptophan (W406) destroys the salt bridge formed with E401. The atomic model is based on the known crystal structure of the corresponding vimentin fragment. The figure is a composite of figures adapted with permission from Journal of molecular biology and Journal of structural biology (110, 131).

Nevertheless, some general features are found in every IF, be it a lamin filament in the nucleus, a desmin filament in a muscle cell, or a keratin filament in an epithelial cell (89). First, all IFs are apolar due to the fact that the principal structural building block within an IF is a tetramer assembled from two antiparallel oriented, dimeric coiled coils. Second, all IFs are highly resistant to extraction with high-ionic-strength buffers, no matter what tissue they are isolated from. This behavior indicates that IFs are held together via an extensive network of both charged and hydrophobic interactions. Third, disease-associated IF protein mutations that affect IF assembly in vitro also disturb IF formation in transfected cells and transgenic animals $(52,90,91)$.

\section{Can atomic structure predict IF structure in vivo?}

A powerful proof of principle as to how predictions derived from both IF protein structure obtained by X-ray crystallography and the behavior of the recombinant protein in vitro translate into IF assembly in living cells has been provided by analysis of vimentin mutated at a specific site in coil 1A. In particular, it was assumed that the bulky tyrosine residue in a " $d$ position" of the heptad within the coil 1A IF-consensus motif would enable the coil $1 \mathrm{~A}$ segment of the rod to open up, probably as a prerequisite for proper assembly $(43,56)$ (Figure 5A). When this residue was replaced with a leucine, which is an optimal " $d$ position" amino acid for forming a coiled coil, crystals of the coil 1A peptide formed bona fide coiled 

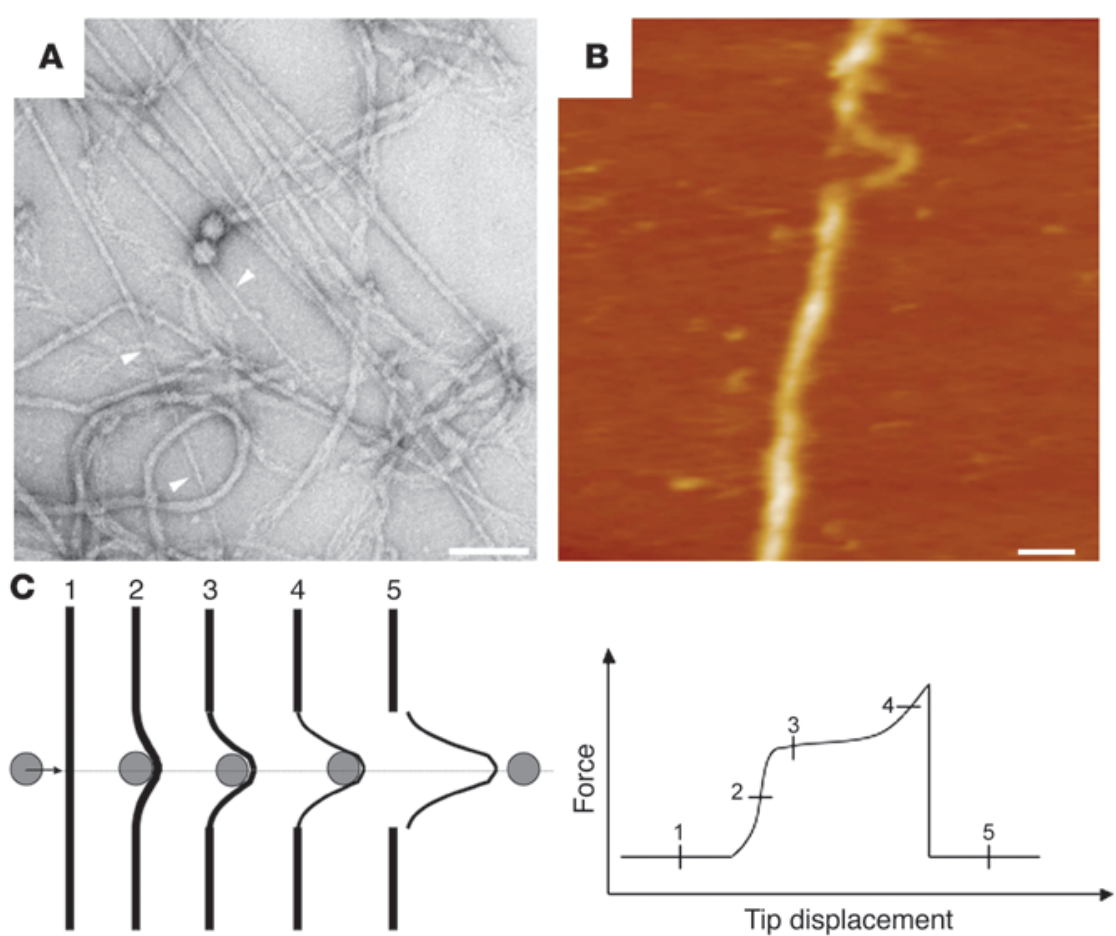

\section{Figure 7}

Tearing and reduction in diameter of mature IFs as observed (A) on an electron microscopic grid and (B) by atomic force microscopy. (C) Schematic description of the filament rupture. The gray disk depicts the atomic force microscopy tip tearing on a filament (black bar). The bottom diagram illustrates the force-displacement curve corresponding to the 5 phases shown in the upper panel. A and $\mathbf{B}$ were reproduced with permission from Journal of molecular biology (125). Scale bars: $100 \mathrm{~nm}$.

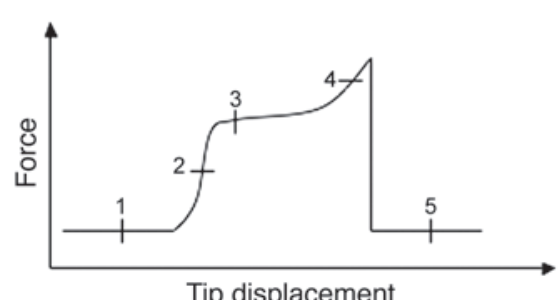

coils (Figure 5B). Compared with the wild-type coil 1A peptide, the mutant peptide exhibited a much higher melting temperature, indicating the formation of strong inter-helical forces (92). Moreover, when the corresponding amino acid change was introduced into full-length vimentin, its in vitro assembly was completely arrested at the ULF stage (83). Upon transfection of this vimentin mutant into vimentin-deficient mouse embryonic fibroblasts, the cells displayed a very distinct, dot-like fluorescent signal pattern indicative of the formation of rather uniform structures (Figure 5C). These dots (Figure 5C, inset) appear to be very similar in size to the vimentin dots described in attaching fibroblasts, which are apparently the in vivo precursors of "squiggles" and short IFs (93, 94), and they may indeed represent ULFs or small aggregates thereof (see Figure 4, phases 1 and 2).

\section{Mutations in IF protein-encoding genes compromise protein function}

At present, 86 distinct human pathologies are known to arise from mutations in IF protein-encoding genes (refs. 4 and 95; and Human Intermediate Filament Database, http://www.interfil. org). The most mutated gene is that encoding human lamin A and lamin C. At present, 330 mutations in this gene have been linked to at least ten different disease entities (96-98). Although, the respective pathomechanisms have remained elusive, several plausible suggestions bearing on their central role as integrators of cellular architecture have been made. These range from interference with resistance of nuclei and whole cells to mechanical stress, to structural alterations in chromatin organization, to modulation of stem cell activities, to changes in gene expression programs and an increase in genomic instability (99-102). Mutations in the genes encoding the epidermal K5 and K14 were the first to be identified to cause disease (reviewed in refs. 50-52). In the basal cell layer of the epidermis, the mutated keratin filaments aggregate heavily and lose their normal connection to des- mosomes and hemidesmosomes. Hence, these mutations in $\mathrm{K} 5$ and K14 interfere with the proper generation of a functional cytoskeleton and, as a consequence, with the stress-absorbing functions of IFs. Both the loss of normal connection to desmosomes and hemidesmosomes and the defect in stress-absorption are evidently central to the tissue fragility observed in individuals carrying these mutations in $\mathrm{K} 5$ and $\mathrm{K} 14$, although other processes, such as signaling and protein turnover, may well be affected and contribute to the pathophysiology (95).

As a consequence, the skin of people born with defective K14 is highly fragile (103). One of the most recent examples of mutations in IF protein-encoding genes that contribute to tissue malfunction relates to the eye lens: a mutation in coil 1B of vimentin, Glu151Lys, has recently been demonstrated to underlie the formation of cataract (104). This glutamic acid is absolutely conserved in vimentins from sharks to humans, and its change to lysine results in a strong kinetic in vitro assembly defect without, however, having a gross effect on the morphology of the mature IF (104).

Desmin mutations affect IF assembly. The muscle-specific IF protein desmin is, in evolutionary terms, rather old, as highly conserved homologs are found in muscle cells from the invertebrate Styela to mammals including humans $(49,105)$. Moreover, all these desmins, including the Styela protein, follow the ULF-type assembly pathway, as demonstrated by in vitro studies with recombinant proteins (27). This conserved assembly behavior indicates that structural and functional aspects coevolved early on, even before vertebrate development started. The first disease-associated mutation in the human desmin-encoding gene was identified about ten years ago (106). Analysis of the effects of the mutation, which led to a deletion of seven amino acids in coil $1 \mathrm{~B}$, demonstrated that it compromised desmin IF assembly both in vitro and in transfected cells. This observation provided a molecular explanation for the huge sarcoplasmic desmin aggregates found in patient muscle and the concomitant severe disturbance in the ordered parallel 
alignment of sarcomeres within individual myofibers. Interestingly, at about the same time, a mutation in the gene encoding a small heat shock protein known as $\alpha B$-crystallin was identified and found to cause a desmin-related myopathy, pointing to a direct functional relationship between desmin networks and these chaperone proteins (107). Ever since, more than 40 desmin disease-associated mutations have been described, and a recent survey of patients with a dilated cardiomyopathy phenotype has revealed desmin to be quite frequently mutated, in approximately $1 \%-2 \%$ of these patients (108).

As the first reported desmin mutant, introduced in the previous paragraph (106), was unable to form regular IFs in vitro but only short irregular filaments, it was of interest to see whether all desmin disease-associated mutations would impair its ability to assemble into bona fide IFs. As expected, with the study of a larger number of desmin disease-associated mutants, a more complex picture emerged: about half of the mutant desmins were able to form extended filaments, whereas the others deviated from the normal assembly pathway at distinct stages by forming various non-IF structures $(109,110)$. Most interestingly, the patient mutations taught structural biologists an important lesson: the introduction of a proline into a central position of the coiled coil does not necessarily impede the ordered organization of the tetramer, ULF, or even the filament. The A360P mutation, which is situated in an a position of the heptad pattern, introduces a change from the typical coiled coil-mediating amino acid alanine to proline, which is supposed to be a "helix breaker." Its occurrence in a coiled coil is therefore highly unusual but, remarkably enough, long regular filaments still formed (Figure 6A). As a result of the mutation, however, the A360P mutant IFs exhibit a diameter of $14.5 \mathrm{~nm}$ as compared with $12.6 \mathrm{~nm}$ for wild-type desmin IFs, and correspondingly harbor 56 subunits per IF cross-section instead of 48 , as determined by mass measurements using scanning transmission electron microscopy (110). Furthermore, the extended filaments observed after 1 hour of in vitro assembly exhibit alterations in their networking ability, indicating a defect in their higher order organization (110). Among the mutants in the second group, which are unable to form extended filaments, some (such as R406W) still associate into regular ULF-like structures. Hence, it is their ability to longitudinally anneal that is affected (Figure $6 \mathrm{~B})$. In the case of R406W, the inter-helical salt bridge formed by the highly conserved aspartate and arginine residues at the end of coil 2B (residues 401 and 406, respectively, in human desmin) is destroyed as a consequence of the mutation (Figure 6C). Some mutant desmins form short IFs that eventually convert into sheet-like assemblies, whereas others convert into small ball-like aggregates (109). These mutants deviate from the normal desmin assembly pathway at distinct steps, indicating they are unable to perform the appropriate subunit reorganizations needed to reach the next assembly stage. Interestingly, mutations located very close to one another within the molecule can give rise to entirely different in vitro assembly phenotypes.

Desmin mutations have an impact on cytoskeletal organization. When transfected into fibroblasts, the two groups of desmin mutants described above (those that form extended filaments and those that cannot) give rise to two distinct cell phenotypes. The filament-forming mutants (e.g., A360P) integrate into the endogenous fibroblast vimentin IF network, whereas the mutants that are unable to assemble into extended filaments (e.g., R406W) completely segregate from the endogenous fibroblast vimentin
IF network (111). Surprisingly, the latter type of desmin mutant causes a "collapse" of the endogenous vimentin IF system onto the cell nucleus. Hence, it may be concluded that the nonintegrating mutant desmin particles compete with vimentin IFs for binding sites with cell-anchoring structures at the cellular periphery (111). Whether similar competition reactions take place in patient muscle and exactly how mutant desmin complexes interfere with the desmin system in myoblasts is presently unknown. Moreover, as observed in patients, all reported myopathic desmin mutations eventually lead to the generation of aggregates that contain mutant desmin, wild-type desmin, and many more proteins of the myocyte cytoskeleton; the molecular mechanism by which the organization of the extra-sarcomeric cytoskeleton is affected by the aggregates is still elusive. Future studies in transgenic animals will surely further our understanding of the differences found in the mechanical properties of individual mutant desmins (112). After all, individual mutations may easily translate into different binding features for cellular cross-bridging factors, membrane components, and organelles such as mitochondria (113).

In the case of the myocyte cytoskeleton, there is further complexity, as it contains IF proteins, such as synemin and syncoilin, that are unable to self-assemble into filaments but need desmin as a scaffold for coassembly, probably by forming heterodimers or higher order complexes with desmin. Hence, mutations in desmin may affect the function of these heteropolymers with strategic components of the myocytes architecture and physiological function. In skeletal muscle, desmin IFs connect to $Z$ bands, costameres, cell nuclei, mitochondria, and desmosomes as well as to other structural proteins within the cytoskeleton. For example, the altered interaction of the desmin mutant E245D with the giant protein nebulin has been reported to negatively influence the length of sarcomeric actin filaments (114). In an analogous manner, the interaction of synemin with components of the extra-sarcomeric cytoskeleton, such as dystrophin, utrophin, talin, and vinculin, may be disturbed by desmin mutations, as the two proteins are in complex within the individual muscle IF, and these alterations may then affect the functional integration of the desmin IF system into the myocyte (115-118). The same scenario may apply to syncoilin and its isoforms that bind to $\alpha$-dystrobrevin (119), although nonstructural functions during regeneration processes have been favored for these IF proteins (120).

\section{Nanomechanical properties of IFs}

In order to arrive at a rational understanding of the altered properties exhibited by mutant IF proteins, more structure determination and more knowledge on the tensile properties of IFs is needed. The IF system provides cells with compliance to small deformations and, at the same time, strengthens cells when large stresses are applied. The molecular basis for the special viscoelastic behavior of IFs has been worked out using rheological methods $(121,122)$. In particular, it was demonstrated that vimentin IFs exhibit a property termed strain stiffening, i.e., upon application of stress the filament suspension becomes more viscoelastic. This property renders IFs distinct from MTs and MFs, which break without a similar response when strain is increased (121). In bulk experiments, it can easily be observed that in vitro application of force to an entire IF network adsorbed to a surface can cause a reduction in the diameter of the single IFs from 12 to $4 \mathrm{~nm}$ (Figure $7 \mathrm{~A})$. Therefore, in order to investigate the tensile properties at the single IF level, atomic force microscopy (AFM) has been employed 
with neurofilaments and desmin IFs (123-125). Using the cantilever of an AFM as a lateral force transducer, it has been determined that an IF may be stretched up to 3.5 fold ( $250 \%$ tensile strain) before it tears (Figure 7, B and C). Interestingly, when monitoring the breaking process, a phase where the filament becomes more viscoelastic in response to the applied strain was clearly visualized (Figure 7C). A future challenge is to investigate the change in cellular plasticity of myocytes that express a mutant desmin and correlate their mechanical properties with the tensile behavior of single IFs assembled from various mutant desmins.

\section{Conclusion}

Based on recent findings of the structural and biophysical properties of IFs, the significance of mutations in IF protein-encoding genes, in terms of their effects on the stability and oligomerization of these coiled-coil proteins, is indeed evident. The next challenge is to understand how these different proteins, which are built according to a common structural blueprint with little sequence homology between distant members of the gene family, translate their distinct sequence patterns into specific functions. For example, how do subtle differences in the hydrophobic seam of the coiled coil, as specified by the amino acid residues occupying the first and fourth positions of the heptad repeats, lead to different stabilities and local changes in the flexibility of the coiled-coil rod domain? Most of the other amino acids, i.e., those residing on the surface of the coiled coil, have the ability to influence interactions within the filament, thereby determining its tensile properties. In addition, some of the amino acids displayed on the filament surface will mediate interactions with other cellular constituents, including the plakins and various chaperones. Because of their distinct primary amino acid sequence, individual IF proteins will differ substantially with respect to their surface properties, hence creating distinct "sites" for binding factors. The relation of mutations to disease severity is surely connected in part to such functions. In a more general sense, in addition to their hard-core structural roles, both cytoplasmic and nuclear IFs may serve as parking platforms for functional proteins such as kinases or transcription factors (126-129). These various functions, in turn, will translate into specific properties, optimizing the plasticity and mechanosensitivity of individual cells within a given tissue.

\section{Acknowledgments}

We thank Rosmarie Sütterlin, Harald Bär, Monika Zwerger, and Norbert Mücke for their help with the figures and Gloria Conover for critical reading of the manuscript. Harald Herrmann acknowledges support from the German Research Foundation (DFG; grant HE 1853) and the German-Israel Foundation. Both Harald Herrmann and Ueli Aebi have been supported by the EURO-Laminopathies research project of the European Commission (contract LSHM-CT-2005-018690). This work was also supported by grants from the National Center of Competence in Research "Nanoscale Science," the Swiss National Science Foundation, and the M.E. Müller Foundation, all awarded to Ueli Aebi. Sergei V. Strelkov and Ueli Aebi acknowledge support from the Swiss Society for Research on Muscular Diseases. Last but not least, Sergei V. Strelkov thanks the Research Council of the Katholieke Universiteit Leuven for support.

Address correspondence to: Harald Herrmann, Group Functional Architecture of the Cell, B065, German Cancer Research Center (DKFZ), D-69120 Heidelberg, Germany. Phone: 49-6221-4235121; Fax: 49-6221-423519; E-mail: h.herrmann@dkfz-heidelberg.de.
1. Steinert, P.M., and Parry, D.A. 1985. Intermediate filaments: conformity and diversity of expression and structure. Annu. Rev. Cell Biol. 1:41-65.

2. Fuchs, E., and Weber, K. 1994. Intermediate filaments: structure, dynamics, function, and disease. Annu. Rev. Biochem. 63:345-382.

3. Lazarides, E. 1980. Intermediate filaments as mechanical integrators of cellular space. Nature. 283:249-256.

4. Szeverenyi, I., et al. 2008. The Human Intermediate Filament Database: comprehensive information on a gene family involved in many human diseases. Hum. Mutat. 29:351-360.

5. Hol, E.M., et al. 2003. Neuronal expression of GFAP in patients with Alzheimer pathology and identification of novel GFAP splice forms. Mol. Psychiatry. 8:786-796.

6. Lazarides, E. 1982. Intermediate filaments: a chemically heterogeneous, developmentally regulated class of proteins. Annu. Rev. Biochem. 51:219-250.

7. Hesse, M., Magin, T.M., and Weber, K. 2001. Genes for intermediate filament proteins and the draft sequence of the human genome: novel keratin genes and a surprisingly high number of pseudogenes related to keratin genes 8 and 18. J. Cell Sci. 114:2569-2575.

8. Herrmann, H., Bar, H., Kreplak, L., Strelkov, S.V., and Aebi, U. 2007. Intermediate filaments: from cell architecture to nanomechanics. Nat. Rev. Mol. Cell Biol. 8:562-573.

9. Kim, S., and Coulombe, P.A. 2007. Intermediate filament scaffolds fulfill mechanical, organizational, and signaling functions in the cytoplasm. Genes Dev. 21:1581-1597.

10. Stuurman, N., Heins, S., and Aebi, U. 1998. Nuclear lamins: their structure, assembly, and interactions.
J. Struct. Biol. 122:42-66.

11. Burke, B., and Stewart, C.L. 2002. Life at the edge: the nuclear envelope and human disease. Nat. Rev. Mol. Cell Biol. 3:575-585.

12. Guelen, L., et al. 2008. Domain organization of human chromosomes revealed by mapping of nuclear lamina interactions. Nature. 453:948-951.

13. Ketema, M., et al. 2007. Requirements for the localization of nesprin- 3 at the nuclear envelope and its interaction with plectin. J. Cell Sci. 120:3384-3394.

14. Heald, R., and McKeon, F. 1990. Mutations of phosphorylation sites in lamin A that prevent nuclear lamina disassembly in mitosis. Cell. 61:579-589.

15. Chou, Y.H., Khuon, S., Herrmann, H., and Goldman, R.D. 2003. Nestin promotes the phosphorylation-dependent disassembly of vimentin intermediate filaments during mitosis. Mol. Biol. Cell. 14:1468-1478.

16. Tsai, M.Y., et al. 2006. A mitotic lamin B matrix induced by RanGTP required for spindle assembly. Science. 311:1887-1893.

17. Downing, K.H., and Nogales, E. 1998. Tubulin and microtubule structure. Curr. Opin. Cell Biol. 10:16-22.

18. Bremer, A., and Aebi, U. 1992. The structure of the F-actin filament and the actin molecule. Curr. Opin. Cell Biol. 4:20-26.

19. Heitlinger, E., et al. 1991. Expression of chicken lamin B2 in Escherichia coli: characterization of its structure, assembly, and molecular interactions. J. Cell Biol. 113:485-495.

20. Jordan, M.A., and Kamath, K. 2007. How do microtubule-targeted drugs work? An overview. Curr. Cancer Drug Targets. 7:730-742.

21. Cooper, J.A. 1987. Effects of cytochalasin and phalloidin on actin. J. Cell Biol. 105:1473-1478.

22. Vale, C., and Botana, L.M. 2008. Marine toxins and the cytoskeleton: okadaic acid and dinophysistoxins. FEBS J. 275:6060-6066.

23. Karabinos, A., Schmidt, H., Harborth, J., Schnabel, R., and Weber, K. 2001. Essential roles for four cytoplasmic intermediate filament proteins in Caenorhabditis elegans development. Proc. Natl. Acad. Sci.U.S. A. 98:7863-7868.

24. Karabinos, A., Schunemann, J., and Weber, K. 2004. Most genes encoding cytoplasmic intermediate filament (IF) proteins of the nematode Caenorhabditis elegans are required in late embryogenesis. Eur. J. Cell Biol. 83:457-468.

25. Karabinos, A., Schulze, E., Schunemann, J., Parry, D.A., and Weber, K. 2003. In vivo and in vitro evidence that the four essential intermediate filament (IF) proteins A1, A2, A3 and B1 of the nematode Caenorhabditis elegans form an obligate heteropolymeric IF system. J. Mol. Biol. 333:307-319.

26. Ding, M., Woo, W.M., and Chisholm, A.D. 2004. The cytoskeleton and epidermal morphogenesis in C. elegans. Exp. Cell Res. 301:84-90.

27. Herrmann, H., Hesse, M., Reichenzeller, M., Aebi, U., and Magin, T.M. 2003. Functional complexity of intermediate filament cytoskeletons: from structure to assembly to gene ablation. Int. Rev. Cytol. 223:83-175.

28. Parry, D.A., and Steinert, P.M. 1999. Intermediate filaments: molecular architecture, assembly, dynamics and polymorphism. Q. Rev. Biophys. 32:99-187.

29. Eckes, B., et al. 1998. Impaired mechanical stability, migration and contractile capacity in vimentindeficient fibroblasts. J. Cell Sci. 111:1897-1907.

30. Eckes, B., et al. 2000. Impaired wound healing in embryonic and adult mice lacking vimentin. J. Cell Sci. 113:2455-2462.

31. Nieminen, M., et al. 2006. Vimentin function in 
lymphocyte adhesion and transcellular migration. Nat. Cell Biol. 8:156-162.

32. Steinert, P.M., and Roop, D.R. 1988. Molecular and cellular biology of intermediate filaments. Annu. Rev. Biochem. 57:593-625.

33. Starger, J.M., Brown, W.E., Goldman, A.E., and Goldman, R.D. 1978. Biochemical and immunological analysis of rapidly purified $10-\mathrm{nm}$ filaments from baby hamster kidney (BHK-21) cells. J. Cell Biol. 78:93-109.

34. Houseweart, M.K., and Cleveland, D.W. 1998. Intermediate filaments and their associated proteins: multiple dynamic personalities. Curr. Opin. Cell Biol. 10:93-101.

35. Parry, D.A. 2005. Microdissection of the sequence and structure of intermediate filament chains. $A d v$. Protein Chem. 70:113-142.

36. Parry, D.A., Strelkov, S.V., Burkhard, P., Aebi, U., and Herrmann, H. 2007. Towards a molecular description of intermediate filament structure and assembly. Exp. Cell Res. 313:2204-2216.

37. Geisler, N., and Weber, K. 1982. The amino acid sequence of chicken muscle desmin provides a common structural model for intermediate filament proteins. EMBO J. 1:1649-1656.

38. Quax-Jeuken, Y.E., Quax, W.J., and Bloemendal, H. 1983. Primary and secondary structure of hamster vimentin predicted from the nucleotide sequence. Proc. Natl. Acad. Sci. U. S. A. 80:3548-3552.

39. Hatzfeld, M., and Weber, K. 1990. The coiled coil of in vitro assembled keratin filaments is a heterodimer of type I and II keratins: use of site-specific mutagenesis and recombinant protein expression. J. Cell Biol. 110:1199-1210.

40. Steinert, P.M. 1990. The two-chain coiled-coil molecule of native epidermal keratin intermediate filaments is a type I-type II heterodimer. J. Biol. Chem. 265:8766-8774.

41. Quax, W., Egberts, W.V., Hendriks, W., Quax-Jeuken, Y., and Bloemendal, H. 1983. The structure of the vimentin gene. Cell. 35:215-223.

42. Herrmann, H., and Aebi, U. 2004. Intermediate filaments: molecular structure, assembly mechanism, and integration into functionally distinct intracellular Scaffolds. Annu. Rev. Biochem. 73:749-789

43. Strelkov, S.V., et al. 2002. Conserved segments 1A and $2 \mathrm{~B}$ of the intermediate filament dimer: their atomic structures and role in filament assembly. EMBO J. 21:1255-1266.

44. Strelkov, S.V., Schumacher, J., Burkhard, P., Aebi, U., and Herrmann, H. 2004. Crystal structure of the human lamin A coil 2B dimer: implications for the head-to-tail association of nuclear lamins. J. Mol. Biol. 343:1067-1080.

45. Zimek, A., and Weber, K. 2002. The gene for a cytoplasmic intermediate filament (IF) protein of the hemichordate Saccoglossus kowalevskii; definition of the unique features of chordate IF proteins. Gene. 288:187-193.

46. Ausmees, N., Kuhn,J.R., and Jacobs-Wagner, C. 2003. The bacterial cytoskeleton: an intermediate filament-like function in cell shape. Cell. 115:705-713.

47. Bagchi, S., Tomenius, H., Belova, L.M., and Ausmees, N. 2008. Intermediate filament-like proteins in bacteria and a cytoskeletal function in Streptomyces. Mol. Microbiol. 70:1037-1050.

48. Herrmann, H., et al. 2000. The intermediate filament protein consensus motif of helix $2 \mathrm{~B}$ : its atomic structure and contribution to assembly. J. Mol. Biol. 298:817-832.

49. Schaffeld, M., Herrmann, H., Schultess, J., and Markl, J. 2001. Vimentin and desmin of a cartilaginous fish, the shark Scyliorhinus stellaris: sequence, expression patterns and in vitro assembly. Eur. J. Cell Biol. 80:692-702.

50. Fuchs, E. 1996. The cytoskeleton and disease: genetic disorders of intermediate filaments. Annu.
Rev. Genet. 30:197-231.

51. Lane, E.B., and McLean, W.H. 2004. Keratins and skin disorders. J. Pathol. 204:355-366.

52. Coulombe, P.A., et al. 1991. Point mutations in human keratin 14 genes of epidermolysis bullosa simplex patients: genetic and functional analyses. Cell. 66:1301-1311.

53. Herrmann, H., Wedig, T., Porter, R.M., Lane, E.B., and Aebi, U. 2002. Characterization of early assembly intermediates of recombinant human keratins. J. Struct. Biol. 137:82-96.

54. Schietke, R., Brohl, D., Wedig, T., Mucke, N., Herrmann, H., and Magin, T.M. 2006. Mutations in vimentin disrupt the cytoskeleton in fibroblasts and delay execution of apoptosis. Eur. J. Cell Biol. 85: $1-10$

55. Hesse, M., et al. 2007. A mutation of keratin 18 within the coil $1 \mathrm{~A}$ consensus motif causes widespread keratin aggregation but cell type-restricted lethality in mice. Exp. Cell Res. 313:3127-3140.

56. Strelkov, S.V., et al. 2001. Divide-and-conquer crystallographic approach towards an atomic structure of intermediate filaments. J. Mol. Biol. 306:773-781.

57. Hut, P.H., et al. 2000. Exempting homologous pseudogene sequences from polymerase chain reaction amplification allows genomic keratin 14 hotspot mutation analysis. J. Invest. Dermatol. 114:616-619.

58. Müller, F.B., et al. 2006. Novel and recurrent mutations in keratin KRT5 and KRT14 genes in epidermolysis bullosa simplex: implications for disease phenotype and keratin filament assembly. Hum. Mutat. 27:719-720.

59. Oh, S.W., Lee, J.S., Kim, M.Y., and Kim, S.C. 2007. Novel keratin 5 mutations in epidermolysis bullosa simplex: cases with unusual genotype-phenotype correlation. J. Dermatol. Sci. 48:229-232.

60. Park, K.Y., et al. 2000. Sporadic cardiac and skeletal myopathy caused by a de novo desmin mutation. Clin. Genet. 57:423-429.

61. Olivé, M., et al. 2004. Desmin-related myopathy: clinical, electrophysiological, radiological, neuropathological and genetic studies. J. Neurol. Sci. 219:125-137.

62. Goudeau, B., et al. 2006. Variable pathogenic potentials of mutations located in the desmin alpha-helical domain. Hum. Mutat. 27:906-913.

63. Sawaishi, Y., Yano, T., Takaku, I., and Takada, G. 2002. Juvenile Alexander disease with a novel mutation in glial fibrillary acidic protein gene. Neurology. 58:1541-1543

64. Ishigaki, K., et al. 2006. TRH therapy in a patient with juvenile Alexander disease. Brain Dev. 28:663-667.

65. Muchir, A., et al. 2000. Identification of mutations in the gene encoding lamins $\mathrm{A} / \mathrm{C}$ in autosomal dominant limb girdle muscular dystrophy with atrioventricular conduction disturbances (LGMD1B). Hum. Mol. Genet. 9:1453-1459.

66. Ki, C.S., et al. 2002. Identification of lamin A/C (LMNA) gene mutations in Korean patients with autosomal dominant Emery-Dreifuss muscular dystrophy and limb-girdle muscular dystrophy $1 \mathrm{~B}$. J. Hum. Genet. 47:225-228.

67. Reichart, B., et al. 2004. Expression and localization of nuclear proteins in autosomal-dominant Emery-Dreifuss muscular dystrophy with LMNA R377H mutation. BMC Cell Biol. 5:12.

68. Taylor, M.R., et al. 2003. Natural history of dilated cardiomyopathy due to lamin $\mathrm{A} / \mathrm{C}$ gene mutations. J. Am. Coll. Cardiol. 41:771-780.

69. Riemer, D., Dodemont, H., and Weber, K. 1993. A nuclear lamin of the nematode Caenorhabditis elegans with unusual structural features; cDNA cloning and gene organization. Eur. J. Cell Biol. 62:214-223.

70. Geisler, N., Schunemann, J., Weber, K., Haner, M., and Aebi, U. 1998. Assembly and architecture of invertebrate cytoplasmic intermediate filaments reconcile features of vertebrate cytoplasmic and nuclear lamin-type intermediate filaments. J. Mol. Biol. 282:601-617.

71. Karabinos, A., Schunemann, J., Meyer, M., Aebi, U., and Weber, K. 2003. The single nuclear lamin of Caenorhabditis elegans forms in vitro stable intermediate filaments and paracrystals with a reduced axial periodicity. J. Mol. Biol. 325:241-247.

72. Foeger, N., et al. 2006. Solubility properties and specific assembly pathways of the B-type lamin from Caenorhabditis elegans. J. Struct. Biol. 155:340-350

73. Wiesel, N., et al. 2008. Laminopathic mutations interfere with the assembly, localization, and dynamics of nuclear lamins. Proc. Natl. Acad. Sci. U. S. A. 105:180-185.

74. Ben-Harush, K., et al. 2009. The supramolecular organization of the $\mathrm{C}$. elegans nuclear lamin filament. J. Mol. Biol. 386:1392-1402.

75. Omary, M.B., Ku, N.O., Tao, G.Z., Toivola, D.M., and Liao, J. 2006. "Heads and tails" of intermediate filament phosphorylation: multiple sites and functional insights. Trends Biochem. Sci. 31:383-394.

76. Dahlstrand, J., Zimmerman, L.B., McKay, R.D., and Lendahl, U. 1992. Characterization of the human nestin gene reveals a close evolutionary relationship to neurofilaments. J. Cell Sci. 103:589-597.

77. Steinert, P.M., et al. 1999. A high molecular weight intermediate filament-associated protein in BHK21 cells is nestin, a type VI intermediate filament protein. Limited co-assembly in vitro to form heteropolymers with type III vimentin and type IV alpha-internexin. J. Biol. Chem. 274:9881-9890.

78. Herrmann, H., et al. 1996. Structure and assembly properties of the intermediate filament protein vimentin: the role of its head, rod and tail domains. J. Mol. Biol. 264:933-953.

79. Mücke, N., et al. 2004. Molecular and biophysical characterization of assembly-starter units of human vimentin. J. Mol. Biol. 340:97-114.

80. Kirmse, R., et al. 2007. A quantitative kinetic model for the in vitro assembly of intermediate filaments from tetrameric vimentin. J. Biol. Chem. 282:18563-18572.

81. Portet, S., et al. 2009. Vimentin intermediate filament formation: in vitro measurement and mathematical modeling of the filament length distribution during assembly. Langmuir. In press.

82. Herrmann, H., and Aebi, U. 1998. Intermediate filament assembly: fibrillogenesis is driven by decisive dimer-dimer interactions. Curr. Opin. Struct. Biol. 8:177-185.

83. Georgakopoulou, S., Möller, D., Sachs, N., Herrmann, H., and Aebi, U. 2009. Near-UV circular dichroism reveals structural transitions of vimentin subunits during intermediate filament assembly. J. Mol. Biol. 386:544-553.

84. Strelkov, S.V., Herrmann, H., and Aebi, U. 2003. Molecular architecture of intermediate filaments. Bioessays. 25:243-251.

85. Inagaki, M., Nishi, Y., Nishizawa, K., Matsuyama, M., and Sato, C. 1987. Site-specific phosphorylation induces disassembly of vimentin filaments in vitro. Nature. 328:649-652.

86. Heins, S., and Aebi, U. 1994. Making heads and tails of intermediate filament assembly, dynamics and networks. Curr. Opin. Cell Biol. 6:25-33.

87. Sihag, R.K., Inagaki, M., Yamaguchi, T., Shea, T.B., and Pant, H.C. 2007. Role of phosphorylation on the structural dynamics and function of types III and IV intermediate filaments. Exp. Cell Res. 313:2098-2109.

88. Moll, R., Franke, W.W., Schiller, D.L., Geiger, B., and Krepler, R. 1982. The catalog of human cytokeratins: patterns of expression in normal epithelia, 'tumors and cultured cells. Cell. 31:11-24.

89. Aebi, U., Häner, M., Troncoso, J., Eichner, R., and Engel, A. 1988. Unifying principles in intermediate 
filament (IF) structure and assembly. Protoplasma. 145:73-81.

90. Albers, K., and Fuchs, E. 1987. The expression of mutant epidermal keratin cDNAs transfected in simple epithelial and squamous cell carcinoma lines. J. Cell Biol. 105:791-806.

91. Albers, K., and Fuchs, E. 1989. Expression of mutant keratin cDNAs in epithelial cells reveals possible mechanisms for initiation and assembly of intermediate filaments. J. Cell Biol. 108:1477-1493.

92. Meier, M., et al. 2009. Vimentin coil $1 \mathrm{~A}-$ a molecular switch involved in the initiation of filament elongation. J. Mol. Biol. In press.

93. Prahlad, V., Yoon, M., Moir, R.D., Vale, R.D., and Goldman, R.D. 1998. Rapid movements of vimentin on microtubule tracks: kinesin-dependent assembly of intermediate filament networks. J. Cell Biol. 143:159-170.

94. Yoon, M., Moir, R.D., Prahlad, V., and Goldman, R.D. 1998. Motile properties of vimentin intermediate filament networks in living cells. J. Cell Biol. 143:147-157.

95. Omary, M.B., Coulombe, P.A., and McLean, W.H. 2004. Intermediate filament proteins and their associated diseases. N. Engl. J. Med. 351:2087-2100.

96. Rankin, J., and Ellard, S. 2006. The laminopathies: a clinical review. Clin. Genet. 70:261-274.

97. Worman, H.J., and Bonne, G. 2007. "Laminopathies": a wide spectrum of human diseases. Exp. Cell Res. 313:2121-2133.

98. Worman, H.J., Fong, L.G., Muchir, A., and Young, S.G. 2009. Laminopathies and the long strange trip from basic cell biology to therapy. J. Clin. Invest. 119:1825-1836.

99. Vlcek, S., and Foisner, R. 2007. Lamins and laminassociated proteins in aging and disease. Curr. Opin. Cell Biol. 19:298-304.

100.Burke, B., and Stewart, C.L. 2006. The laminopathies: the functional architecture of the nucleus and its contribution to disease. Annu. Rev. Genomics Hum. Genet. 7:369-405.

101. Dechat, T., et al. 2007. Alterations in mitosis and cell cycle progression caused by a mutant lamin A known to accelerate human aging. Proc. Natl. Acad. Sci. U. S. A. 104:4955-4960.

102.Dechat, T., et al. 2008. Nuclear lamins: major factors in the structural organization and function of the nucleus and chromatin. Genes Dev. 22:832-853.

103. Chan, Y., et al. 1994. A human keratin 14 "knockout": the absence of K14 leads to severe epidermolysis bullosa simplex and a function for an intermediate filament protein. Genes Dev. 8:2574-2587.
104.Müller, M., et al. 2009. Dominant cataract formation in association with a vimentin assembly disrupting mutation. Hum. Mol. Genet. 18:1052-1057.

105. Riemer, D., and Weber, K. 1998. Common and variant properties of intermediate filament proteins from lower chordates and vertebrates; two proteins from the tunicate Styela and the identification of a type III homologue. J. Cell Sci. 111:2967-2975.

106.Munoz-Marmol, A.M., et al. 1998. A dysfunctional desmin mutation in a patient with severe generalized myopathy. Proc. Natl. Acad. Sci. U. S. A. 95:11312-11317.

107. Vicart, P., et al. 1998. A missense mutation in the alphaB-crystallin chaperone gene causes a desminrelated myopathy. Nat. Genet. 20:92-95.

108.Taylor, M.R., et al. 2007. Prevalence of desmin mutations in dilated cardiomyopathy. Circulation. 115:1244-1251.

109.Bär, H., et al. 2005. Severe muscle disease-causing desmin mutations interfere with in vitro filament assembly at distinct stages. Proc. Natl. Acad. Sci.U.S. A. 102:15099-15104.

110. Bär, H., et al. 2006. Impact of disease mutations on the desmin filament assembly process. J. Mol. Biol. 360:1031-1042.

111.Bär, H., et al. 2006. Forced expression of desmin and desmin mutants in cultured cells: impact of myopathic missense mutations in the central coiled-coil domain on network formation. Exp. Cell Res. 312:1554-1565.

112.Shah, S.B., et al. 2004. Structural and functional roles of desmin in mouse skeletal muscle during passive deformation. Biophys. J. 86:2993-3008.

113. Capetanaki, Y., et al. 2007. Muscle intermediate filaments and their links to membranes and membranous organelles. Exp. Cell Res. 313:2063-2076.

114.Conover, G.M., Henderson, S.N., and Gregorio, C.C. 2009. A myopathy-linked desmin mutation perturbs striated muscle actin filament architecture. Mol. Biol. Cell. 20:834-845.

115.Titeux, M., et al. 2001. Human synemin gene generates splice variants encoding two distinct intermediate filament proteins. Eur. J. Biochem. 268:6435-6449.

116.Bhosle, R.C., Michele, D.E., Campbell, K.P., Li, Z., and Robson, R.M. 2006. Interactions of intermediate filament protein synemin with dystrophin and utrophin. Biochem. Biophys. Res. Commun. 346:768-777.

117. Sun, N., Critchley, D.R., Paulin, D., Li, Z., and Robson, R.M. 2008. Identification of a repeated domain within mammalian alpha-synemin that interacts directly with talin. Exp. Cell Res. 314:1839-1849.

118.Sun, N., Critchley, D.R., Paulin, D., Li, Z., and Robson, R.M. 2008. Human alpha-synemin interacts directly with vinculin and metavinculin. Biochem. J. 409:657-667.

119.Zhang, J., et al. 2008. Syncoilin is required for generating maximum isometric stress in skeletal muscle but dispensable for muscle cytoarchitecture. Am. J. Physiol. Cell Physiol. 294:C1175-C1182.

120.Kemp, M.W., et al. 2009. Syncoilin isoform organization and differential expression in murine striated muscle. J. Struct. Biol. 165:196-203.

121.Janmey, P.A., Euteneuer, U., Traub, P., and Schliwa, M. 1991. Viscoelastic properties of vimentin compared with other filamentous biopolymer networks. J. Cell Biol. 113:155-160.

122.Wagner, O.I., et al. 2007. Softness, strength and self-repair in intermediate filament networks. Exp. Cell Res. 313:2228-2235.

123. Guzman, C., et al. 2006. Exploring the mechanical properties of single vimentin intermediate filaments by atomic force microscopy. J. Mol. Biol. 360:623-630.

124.Kreplak, L., Herrmann, H., and Aebi, U. 2008. Tensile properties of single desmin intermediate filaments. Biophys. J. 94:2790-2799.

125.Kreplak, L., Bär, H., Leterrier, J.F., Herrmann, H., and Aebi, U. 2005. Exploring the mechanical behavior of single intermediate filaments. J. Mol. Biol. 354:569-577.

126.Heessen, S., and Fornerod, M. 2007. The inner nuclear envelope as a transcription factor resting place. EMBO Rep. 8:914-919.

127.Ivaska, J., Pallari, H.M., Nevo, J., and Eriksson, J.E. 2007. Novel functions of vimentin in cell adhesion, migration, and signaling. Exp. Cell Res. 313:2050-2062.

128. Han, X., et al. 2008. Tethering by lamin A stabilizes and targets the ING1 tumour suppressor. Nat. Cell Biol. 10:1333-1340.

129.Gonzalez, J.M., Navarro-Puche, A., Casar, B., Crespo, P., and Andres, V. 2008. Fast regulation of AP-1 activity through interaction of lamin A/C, ERK1/2, and c-Fos at the nuclear envelope. J. Cell Biol. 183:653-666.

130.Colucci-Guyon, E., et al. 1994. Mice lacking vimentin develop and reproduce without an obvious phenotype. Cell. 79:679-694.

131.Bär, H., Strelkov, S.V., Sjöberg, G., Aebi, U., and Herrmann, H. 2004. The biology of desmin filaments: how do mutations affect their structure, assembly, and organisation? J. Struct Biol. 148:137-152. 